\title{
Operationalizing the Nature Futures Framework to catalyze the development of nature-future scenarios
}

\author{
Carolyn Lundquist $^{1,2} \cdot$ Shizuka Hashimoto $^{3} \cdot$ Mekuria Argaw Denboba $^{4} \cdot$ Garry Peterson $^{5} \cdot$ Laura Pereira $^{5,6}$. \\ Dolors Armenteras $^{7}$
}

(c) Springer Japan KK, part of Springer Nature 2021

\section{Introduction}

Scenarios and models are powerful tools for exploring the likely future outcomes of alternative social-ecological development pathways and for informing policy decisions. However, one of the key messages made by the Methodological Assessment of Scenarios and Models by the Intergovernmental Platform on Biodiversity and Ecosystem Services (IPBES) was that existing scenarios of biodiversity and ecosystem services have important shortcomings and gaps that constrain their usefulness for halting the dangerously declining trend of nature and Nature's Contributions to People (NCP) (IPBES 2016b). Specifically, most of the existing scenarios and related analyses, especially at global and regional scales, are limited to assessing the impacts of drivers on a few facets of nature and NCP, often failing to address their linkages or feedbacks, to consider multiple spatial scales, or to incorporate policy objectives related to nature conservation (IPBES 2016b, 2019). Besides, they have limitations in their ability to incorporate shared values, norms, and policy objectives related to nature conservation and good quality of life (IPBES 2016a). Most importantly,

Shizuka Hashimoto

ahash@g.ecc.u-tokyo.ac.jp

Carolyn Lundquist

Carolyn.Lundquist@niwa.co.nz

Mekuria Argaw Denboba

mekuria.argaw@aau.edu.et

Garry Peterson

garry.peterson@su.se

Laura Pereira

laura.pereira@su.se

Dolors Armenteras

darmenterasp@unal.edu.co

1 School of Environment, The University of Auckland, Auckland, New Zealand the existing approaches often emphasize negative trends and drivers, rather than identifying desirable futures for nature and people with multiple alternative pathways to reach there.

To address these shortcomings and to build on the recommendations made by the Methodological Assessment of Scenarios and Models (IPBES 2016b), IPBES Plenary formed an expert group (2016-2019) and then a task force (2019-present) on scenarios and models to catalyze the development of nature-future multi-scale scenarios. The former IPBES scenarios and models expert group set out a research strategy to address some of the key messages that emerged in the assessment and initiated the development of a new framework to catalyze the development of desirable, multiscale scenarios for nature to explore pathways that can enable humanity to meet the desired 2050 Vision for Biodiversity under the Convention on Biological Diversity of 'Living in harmony with nature.' The key developments required for novel scenarios include better integration of the dynamic social-ecological feedbacks between nature and good quality of life, recognition, and inclusion of both synergies and trade-offs between nature and Sustainable Development Goals, the use of participatory approaches,

2 National Institute of Water and Atmospheric Research, Hamilton, New Zealand

3 Department of Ecosystem Studies, University of Tokyo, Tokyo, Japan

4 Center for Environmental Science, Addis Ababa University, Addis Ababa, Ethiopia

5 Stockholm Resilience Centre, Stockholm University, Stockholm, Sweden

6 Global Change Institute, Wits University, Johannesburg, South Africa

7 Department of Biology, Universidad Nacional de Colombia, Bogotá, Colombia 
and the ability to recognize a multiplicity of pathways and pluralistic shared-value perspectives for desirable and sustainable futures for nature and people (IPBES 2016b; Rosa et al. 2017, 2020; Pereira et al. 2020).

This resulted in the development of the Nature Futures Framework (NFF), a heuristic tool that helps users to visualize and describe diverse pathways of desirable futures to slow and reverse the declining trend of nature and NCPs (Pereira et al. 2020). The NFF is based on an in-depth analysis of a wide range of visions of positive futures for biodiversity and people (Lundquist et al. 2017; Pereira et al. 2020) and embraces the diversity of human-nature relationships that reflect three primary value perspectives of nature (i.e., intrinsic, instrumental, and relational). Pereira et al. (2020) documented the iterative process that was undertaken by the IPBES scenarios and models expert group from 2016 to 2019 from which the Nature Futures Framework emerged. The new scenario framework, placing the human-nature relationship at its core, facilitates the integration of interlinkages of social-ecological systems across direct and indirect drivers, nature, NCP, and Good Quality of Life (GQL), and the incorporation of multiple systems of knowledge across scales and sectors, which is in line with the IPBES conceptual framework. By doing so, the NFF enables scenarios to shift from the classic ways of projecting impacts of society on nature to desirable nature-future visions and pathways. Moreover, this framework allows those involved in future scenario building to recognize and address plural and sometimes conflicting values ascribed to nature and NCP in a more explicit manner, which conventional scenario building methods (e.g., scenario-axes technique) often fail to capture. They can also reflect indigenous and local knowledge systems and values, socio-cultural contexts, alternative governance systems, sustainable resource utilization, and conservation of nature if applied appropriately. To date, IPBES has been supporting work to assist in translating the NFF into quantitative and qualitative scenarios to facilitate the development of locally or regionally specific scenarios that apply the broad and flexible descriptions provided in the NFF to a diversity of settings.

\section{Aims and scope of special feature}

This special feature (SF) will present a variety of papers on the Nature Futures Framework. These papers will explore, deepen, and apply the Nature Futures Framework across a variety of contexts and scales to support ongoing and future IPBES assessments. In particular, we welcome contributions that address the following themes:

- Development of methodological guidance and illustrative narratives to support the use of the NFF for developing scenarios for nature and people at multiple scales
- Application of the NFF in specific geographic/ecosystem contexts including urban and rural landscapes, freshwater systems, coastal areas, and the high seas

- Application of the NFF in thematic/topical contexts such as invasive alien species and sustainable use of wild species

- Application of the NFF to Shared Socioeconomic Pathway (SSP) scenarios and impact modeling of Sustainable Development Goals (SDGs) and Post-2020 Biodiversity Framework targets

- Application of the NFF to consider SDGs/Convention on Biological Diversity (CBD) indicators to explore synergies and trade-offs amongst multiple goals/targets of the Post-2020 Biodiversity Framework

- Application of the NFF to model drivers of transformative change (qualitative-quantitative linkages including Indigenous and local knowledge.

- Application of the innovative modeling approaches (e.g., agent-based models) to describe the role of social-ecological feedbacks in the NFF.

- Reflection on the NFFs complementarity to existing concepts/value frameworks and how the NFF can complement existing scenario-building frameworks?

\section{Deadline, submission, and review process}

This Special Feature (SF) is dedicated to multidisciplinary topics with a broad scope and aims to publish a low number of largely invited articles. We encourage authors to submit extended abstracts (maximum 500 words) to the SF editors through the abstract submission form (see below URL). We will invite a selection of authors to submit fulllength manuscripts through the journal's electronic editorial management system. Authors are required to follow the publisher's formatting guidelines and length limitations as per the guideline. At this point, authors should state if they are submitting their work to be considered for the "Operationalizing the Nature Futures Framework to catalyze the development of nature-future scenarios."

- Submit abstracts from https://forms.gle/hJUc6amg8g s8gkf3A

- Deadline for abstract submission: 30 September 2021

- Selection of abstracts by the task force: 31 October 2021

- Deadline for full paper submission: 30 April 2022

\section{Authors guidelines}

http://www.springer.com/environment/environmental+ management/journal/11625?detailsPage $=$ pltci_728046. 


\section{Submission guidelines}

For submission through the EM system, please register in the EM system (below link) and submit your article by selecting the SF title. You can see the author tutorial on the right side of the registration page. Please tag your submission with the SF tag "Operationalizing the Nature Futures Framework to catalyze the development of nature-future scenarios."

http://www.editorialmanager.com/sust/mainpage.html.

\section{References}

IPBES (2016a) The methodological assessment report on scenarios and models of biodiversity and ecosystem services. IPBES

IPBES (2016b) Summary for policymakers of the methodological assessment of scenarios and models of biodiversity and ecosystem services of the Intergovernmental Science-Policy Platform on Biodiversity and Ecosystem Services. IPBES
IPBES (2019) Summary for policymakers of the global assessment report on biodiversity and ecosystem services of the Intergovernmental Science-Policy Platform on Biodiversity and Ecosystem Services. IPBES

Lundquist CJ, Pereira HM, Alkemade JRM, et al (2017) Visions for nature and nature's contributions to people for the 21st century : Report from an IPBES visioning workshop held on 4-8 September 2017 in Auckland, New Zealand. NIWA

Pereira LM, Davies KK, Belder E et al (2020) Developing multiscale and integrative nature-people scenarios using the Nature Futures Framework. People and Nature 13:1

Rosa I, Pereira HM, Ferrier S et al (2017) Multiscale scenarios for nature futures. Nat Ecol Evol 1:1416-1419

Rosa I, Purvis A, Alkemade R et al (2020) Challenges in producing policy-relevant global scenarios of biodiversity and ecosystem services. Global Ecol Conservation 22:00886

Publisher's Note Springer Nature remains neutral with regard to jurisdictional claims in published maps and institutional affiliations. 